\title{
Towards Collaboration-Centric Pattern-Based Software Development Support
}

\author{
Christoph Dorn \\ Distributed Systems Group \\ Vienna University of Technology, Austria \\ dorn@infosys.tuwien.ac.at
}

\author{
Alexander Egyed \\ Institute for Systems Engineering and Automation \\ Johannes Kepler University, Linz, Austria \\ alexander.egyed@jku.at
}

\begin{abstract}
Software engineering activities tend to be loosely coupled to allow for flexibly reacting to unforeseen development complexity, requirements changes, and progress delays. This flexibility comes a the price of hidden dependencies among design and code artifacts that make it difficult or even impossible to assess change impact. Incorrect change propagation subsequently results in costly errors. This position paper proposes a novel approach based on monitoring engineering activities for subsequent high-level pattern detection. Patterns of (i) collaboration structures, (ii) temporal action sequences, and (iii) artifact consistency constraints serve as input to recommendation and automatic reconfiguration algorithms for ultimately avoiding and correcting artifact inconsistencies.
\end{abstract}

Index Terms-monitoring, pattern detection, software engineering, recommendation, collaboration structures

\section{INTRODUCTION}

Software development consists of design and development steps typically performed by multiple software engineers, using heterogeneous tools, models, and expertise to support requirements capture, design, implementation, testing, debugging, bug reporting and many other activities. Such activities tend to be loosely coupled for two main reasons: (i) artifacts such as requirements, pieces of code, or test scenarios are created and manipulated by separate engineers potentially even using different tools and (ii) dealing with changing requirements, unforeseen complexity, and progress delays during the development process requires a high degree of flexibility for developer coordination and communication.

This results in fragmentation where individual engineers are perhaps aware of their particular work focus but lack a more comprehensive overview. It is quite rare to explicitly describe how various development artifacts depend on one another (e.g., traceability [1]). It is virtually never described how or why these artifacts came about and who created them using what other artifacts as input. Subsequently, dependencies among development artifacts remain implicit (i.e., they tend to remain in the heads of engineers if they are discovered at all). This is particularly a problem when changes occur because the lack of explicit dependencies makes it nearly impossible to understand how changes affect the development artifacts. For example, a requirement change should not only trigger design/code changes but also changes to test scenarios, documentation, and

This work is supported in part by the Austrian Science Fund (FWF) under grant number J3068-N23. other artifacts - artifacts which likely were created by different engineers/stakeholders using different tools at different times. A change then should trigger collaboration among software engineers to update these artifacts. Not understanding or perceiving the need of such collaborations is then the root cause for failure to correctly propagate changes and hence the root cause for inconsistencies among development artifacts leading to costly rework and even project failure [2].

Our goal is supporting software engineers in perceiving required collaboration, dependencies among artifacts, and suitable actions by means of recommendations and automatic reconfigurations of the software development environment. To this end, we propose recording the actions of software engineers, deducing patterns from that information, and exploiting them for recommendations. We aim at identifying patterns that reflect three dependencies types: structural collaboration patterns among developers, tasks, and artifact (e.g., what artifacts do developers read/manipulate and how do they coordinate), temporal activity patterns (e.g., which sequences of artifact reading, manipulating, and communication exist), and artifact dependency patterns (e.g., what relationships exist among the artifacts - consistency, traceability, etc.). These patterns then form that basis for adaptation and recommendation techniques that are aware of the arising structural implications. The following Section II motivates this approach based on an illustrative example, followed by our detailed approach in Section III and comparison to related work in Section IV.

\section{Motivating Example}

Suppose a software development team utilizing dedicated tools for requirement elicitation, architecture design, code versioning, bugtracking, and an IDE for source code development. Additionally team members also apply instant messaging, email, and document sharing to coordinate their work (see Fig. 1 bottom).

At some stage, the team needs to handle a changing requirement demanding a functionality adjustment. Consequently, the architecture and certain components need analysis to assess the changed requirement's impact (i.e., identifying architectural changes that satisfy the changed requirement, if any). Subsequently identifying affected design artifacts and source code files, determining necessary tasks to maintain consistency with other related artifacts, and selecting a suitable set of 
developers with the necessary in-depth knowledge and skills is a tremendous, error prone task. It requires developers to remember how they implemented the now-changed requirement in the past (who previously designed, implemented, tested it) to appropriately change all affected artifacts.

For this scenario, how can we support the system architect, when she receives no notification on requirement updates? How can we determine when architecture changes indirectly affect source code artifacts? How can the software architect detect that an affected code artifact has no longer a responsible developer and who might be a suitable replacement? In the next section we present our approach for a framework addressing such questions.

\section{APPROACH}

Mechanisms and techniques for supporting the evolution of software development artifacts and developer collaborations establish a tightly integrated feedback loop comprising steps for bottom-up activity monitoring, pattern matching and analysis, and subsequent recommendation and reconfiguration (Fig. 1).

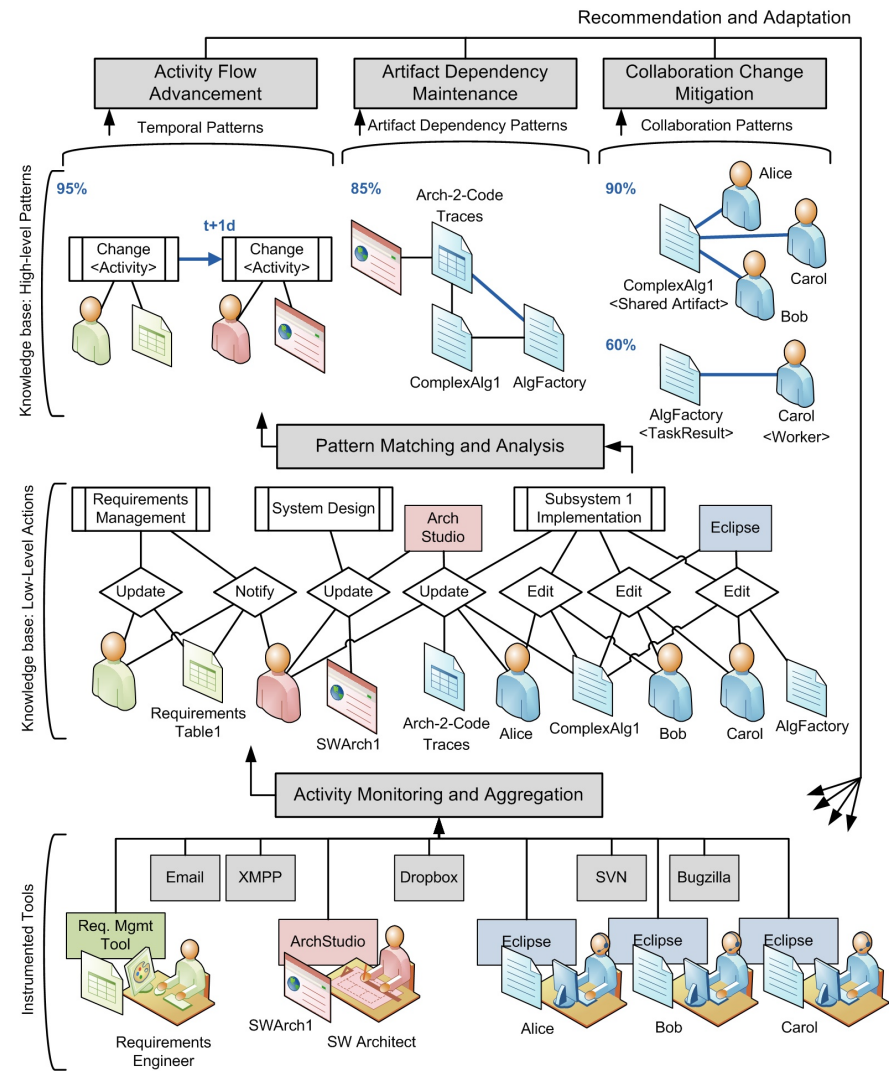

Fig. 1. Approach.

\section{A. Activity Monitoring and Processing}

Observation of developer actions is central to many previous approaches (e.g., ). We plan to extend the scope of monitoring beyond source code production within IDEs to include manipulating of design artifacts as well as communication among developers. Our approach foresees instrumenting tools such as those from the scenario above. We essentially record which developer is manipulating what artifacts and, while doing so, communicates with which other developers.

Activity Monitoring and Aggregation takes the individual actions, filters out excessive actions, correlates actions (e.g., identifying the same artifact in the SVN and within an IDE), and resolves uncertainties. The Low-Level Actions are stored in the Knowledge base (see Fig. 1 center) as a composition of actions observed. The basic structure of an action is one that ties together an engineer with an artifact, an activity, and a tool. For example, Alice added a method to the ComplexAlg1 source file using Eclipse. These tiny fragments are merged together into a connected network of low-level actions. For example, we can then see who else also edited ComplexAlg1 regardless of their used tool or activity context.

\section{B. Pattern Matching and Analysis}

The low-level view serves as the input to three types of Pattern Matching and Analysis heuristics which extract highlevel dependencies and structures among engineers and artifacts. A heuristic focusing on temporal dependencies detects multiple occurrences of following example event sequence: \{an requirement engineer edits the requirement description, followed by an email to the software architect, and the architecture subsequently updating the architecture model and concludes that a requirement change is followed by an architecture change. Multiple, interleaving edits on code file ComplexAlgorithm1 by developers Alice, Bob, and Carol lead a collaboration pattern heuristic to the conclusion that the file is worked upon as a shared artifact rather than a task specifically assigned to Alice (with Bob and Carol merely giving advice). Given a set of consistency constraints (e.g., each architecture component must map to at least one source code artifact) and a set of immediate architecture-to-code mappings, an artifact dependency heuristic may analyze software source code dependencies and tag supporting code as relevant to a particular architecture element. Subsequent analysis calculates pattern-specific properties needed by recommendation and adaptation algorithms (e.g., update rate for a shared artifact, average duration between changing code and running regression tests) before the high-level patterns are stored in the Knowledge base in form of likely relations, dependencies, and constraints among developers, tasks, and artifacts (Fig. 1 upper half).

\section{Recommendation and Adaptation}

Rather than having to deal with low-level individual artifact editing actions, email communication, and task assignments, high-level patterns provide the basis for algorithms that ultimately deliver user recommendations and instrument reconfigurations autonomously when a change occurs (Fig. 1 top). Such changes describe high-level pattern events (e.g., new pattern instances, updated pattern properties) as well as lowlevel actions (e.g., a file has been changed). In our scenario, a combination of three mechanisms assists the engineering 
team in the following ways. Activity Flow Advancement exploits detected temporal patterns. In our scenario it will notify the software architect of the exact requirement change and the affected architecture elements if it observes neither communication between the requirements engineer and the architect nor read or write access to the affected architecture elements within a given time frame. Without such notification the system architect may remain unaware of changes made by the requirements engineer.

Once the system architect identifies (directly and indirectly) affected components and connectors, Artifact Dependency Maintenance identifies the location and cause for potential artifact inconsistencies. Here, it uses the artifact consistency patterns to determine the respective primary and secondary source code artifacts (here: ComplexAlg1, AlgFactory).

Collaboration Change Mitigation assists in determining the most suited developers based on their involvement in source code authoring for reflecting the architecture changes in code artifacts. This mechanism generally deals with undesirable team-topology situations (e.g., code without responsible developer). Taking the underlying detected collaboration patterns into consideration, we may recommend that Carol should take over sole responsibility for ComplexAlg1 and AlgFactory and subsequently carry out the change propagation.

While recommendations certainly present a low-invasive technique to support a software developer team, our approach foresees also autonomous reconfigurations. Example adaptations include automatically annotating model and code artifacts with warnings and information of the ongoing change, creating a chat room linked to the relevant artifacts and developers, and even assigning change propagation tasks to particular engineers.

\section{Discussion}

We are of the opinion that tool integration is key for supporting co-evolution of software development artifacts and collaboration structures (rather than limiting ourselves to nonintrusive observation of user actions via SVN logs or email repositories). Thus, initially, there will be a trade-off between (a) achieving high precision and extend of the collaboration structures and artifact dependencies that are automatically observable, and (b) simultaneously keeping the burden onto the developer minimal. Yet, an increase in the number of integrated tools, the level of observable detail, and the number of participating developers will enable a more complete, meaningful view. Over time, this will lower the developer's burden while raising the perceived benefit. Note, however, that there will always be an element of uncertainty in the artifacts dependencies and collaboration structures we observe. These are due to:

- Incomplete observations: Ideally we would like to capture complete artifact dependencies and collaboration structures. Yet, it is likely infeasible to obtain complete developer actions. Some information communication may be non-accessible or incomprehensible (e.g., an oral communication) and must be disregarded. Even communication and manipulations involving a tool can be problematic. Tools by third party vendors typically offer only limited monitoring capabilities. Thus only parts of all development actions are available for reasoning and recommendation.

- Context switching: A core assumption is that artifacts investigated and manipulated concurrently are likely dependent on one another [3]. However, developers typically do switch between different strands of work dynamically and engage in frequent short-term side-tracking from their main activity/goal.

While it may thus be impossible to completely eliminate the possibility of falsely identified dependencies, following mechanisms address the inherent uncertainty:

- delayed uncertainty resolving: during live editing we understand temporal dependencies among artifacts and developers but a latter, fine-grained diffing of artifacts pre-post state may reveal in more level of detail what exactly was changed.

- continuous pattern refinement: incoming actions allow for better pattern detection thus enabling refinement or even revocation of pattern instances.

- probabilistic pattern instantiation: detection algorithms assign probabilities to pattern instantiations and pattern elements to express uncertainty and thus allowing recommendation algorithms to trigger only for specified reliability thresholds.

\section{RElated Work}

\section{Collaboration Awareness}

In Software Engineering environments, artifacts replace activities as the main, explicit element for collaboration and coordination (merely generic tasks for work assignment remain). Most development awareness tools address the code implementation and maintenance phase; occasionally also the testing phase. Their primary focus is typically on change management (e.g., ELVIN/Tickertape [4], SoftCHANGE [5]), conflict avoidance (e.g., Palantir [6], Codebook [7], RaisAware [8]), and understanding developer activities (e.g., FASTDash: [9], Jazz [10], Ariadne [11], Tesseract [12], ProxiScientia [13]). Figueiredo and de Souza [14] extend change impact analysis to design artifacts using traceability links. These tools have several aspects in common. All either lack near-real time information when relying on code and communication repositories as data source and/or provide near-real time awareness but remain restricted to a single (stand-alone or integrated) tool. Awareness information typically consists of direct relations among artifacts and developers (e.g., related developers and artifacts for a particular artifact) at the source code level, thereby remaining unaware of consistency and traceability concerns with design artifacts. Relevance is derived from an aggregated set of low-level data (e.g., from source code commit events, code read/write events, documentation, issue tracking, and change management systems) without extraction of high-level patterns.

\section{Coordination Requirements}

Based on Conway's insights [15], socio-technical congruence (STC) [16] measures the extent of developer coordination capabilities meeting the underlying work coordination requirements. Several research efforts apply social network analysis 
and data-mining [17], [18], [19] for investigating coordination issues in software development teams. While STC mostly focuses on alignment (or lack thereof) of task dependencies with developer communication links, Jiang et al. [20] introduce also knowledge-centric and resource-centric congruence. Doing so, existing approaches remain unaware of different dependency types [21] and corresponding coordination mechanisms [22]. We propose high-level patterns for better addressing a team's various coordination needs. We thereby aim beyond mining task routing patterns (e.g., [23]) which remain artifact unaware.

\section{Adaptation and Recommendation}

Recommendation mechanisms in CWEs and software development projects typically determine a set of relevant people (Ensemble [24]), expertise, and artifacts (code, bug reports, documentation) for the situation at hand. Relevance arises from project repositories via topic searches and filters (Expertise Recommender [25]), task involvement and development activities (Hipikat/Mylar [26]), frequently or subsequently visited code (Team Tracks [3]), developer activity similarity (Proximity [27]), general user activity and interaction [28], and access to similar artifacts, including artifact similarity [29]. The later works provide more sophisticated recommendations as they consider more than only immediate relations (see also collaboration awareness tools above). In general, recommendations remain limited to listing/ranking users or artifacts. To the best of knowledge, no tools exist that recommend steps or tasks for the underlying situation, much less autonomously carry out support activities and collaboration reconfigurations.

\section{Flexible process systems}

Software development-focused process support takes on various forms. On the micro-level, Zhao et al. apply Little-JIL for describing fine-grained steps involved in refactoring [30]. Little-JIL provides also the basis for a provenance framework to help developers track artifact dependencies during rework [31]. While this approach captures which artifacts where changed by what rework activity, the involved users and internal dependencies remain implicit. Hebig et al. [32] describe how various software design and code artifacts dependencies emerge from MDE activities such as generation, transformation, mapping, and manual editing. Process support at the macro-level assumes pre-defined process models and rigorous tool integration. Kedji et al. provide a collaborationcentric development process model and corresponding DSL [33]; Moser et al. [34] build an engineering bus for integration of development activities for flexible industrial automation systems. These micro and macro-level approaches are software development artifact aware but lack the required flexibility.

Artifact Dependencies, Inconsistency Management, and Change Impact

In context of design models, change impact analysis is in its infancy. Most progress has been on the detecting of inconsistencies [35], [36], [37], [38], [39], [40], [41], [42] because an inconsistency is indicative of an incomplete or incorrect change propagation. There also have been attempts in generating fixes for inconsistencies [43], [44], [37] where the "fixes" could be seen analogous to propagated changes. Approaches such as xLinkIt [43] or Beanbag [37] are representative of this group. Change propagation has been addressed more directly by Briand et al. [45] who identify specific change propagation rules. However, there is generally no guarantee of correctness associated with change propagation (which is indicative of the challenges we are addressing and the heuristical nature we propose). The problem of change propagation is analogous to the quite extensive works on model transformation [46] and code generators. Transformations are quite useful in very specialized domains where one model can be derived from another model (partially or completely). However, our proposed work is meant to be useful for models (or model elements) that cannot be derived from other models (or model elements). Therefore, transformation alone is insufficient to solve our problem [47]. Instead, this proposed work will reason about restrictions imposed by constraints and other patterns - a problem that is analogous to constraint-based reasoning (e.g., SAT solving technology [48]) however optimized specifically towards the domain of modeling.

\section{Conclusions}

Extracting high-level patterns for software development recommendation and adaptation constitutes a great challenge due to inherent collaboration dynamics and uncertainty but also promised great benefit. Our upcoming research activities focus primarily on the technical framework and mechanisms ultimately aimed at significantly reducing inconsistent and/or incomplete change propagation. In the course of our research two main empirical research questions of high significance will then arise: (i) how precise and comprehensive do we have to document developer actions, artifacts, and their relations and (ii) how extensive and invasive may our techniques be when dynamically reconfiguring a developer team, for example, to ensure that the most suitable developer available is involved in a change task.

\section{REFERENCES}

[1] O. Gotel and A. Finkelstein, "Extended requirements traceability: Results of an industrial case study," in 3rd IEEE International Symposium on Requirements Engineering (RE'97), January 5-8, 1997, Annapolis, MD, USA, 1997, pp. 169-.

[2] B. W. Boehm, Software Engineering Economics. Englewood Cliffs, NJ: Prentice Hall, 1981.

[3] R. DeLine, M. Czerwinski, and G. Robertson, "Easing program comprehension by sharing navigation data," in Proc. of the IEEE Symp. on Visual Languages and Human-Centric Computing, VLHCC '05. Washington, DC, USA: IEEE Computer Society, 2005, pp. 241-248.

[4] G. Fitzpatrick, P. Marshall, and A. Phillips, "Cvs integration with notification and chat: lightweight software team collaboration," in Proceedings of the 20th Conf. on Computer Supported Cooperative Work, CSCW '06. New York, NY, USA: ACM, 2006, pp. 49-58.

[5] D. M. German, A. Hindle, and N. Jordan, "Visualizing the evolution of software using softChange," International Journal of Software Engineering and Knowledge Engineering, vol. 16, no. 1, pp. 5-21, 2006.

[6] A. Sarma, Z. Noroozi, and A. van der Hoek, "Palantir: Raising awareness among configuration management workspaces," International Conference on Software Engineering, p. 444, 2003.

[7] A. Begel, Y. P. Khoo, and T. Zimmermann, "Codebook: discovering and exploiting relationships in software repositories," in $\operatorname{ICSE}(1), 2010$, pp. $125-134$.

[8] J. M. dos Reis Costa, R. M. Feitosa, and C. R. B. de Souza, "Tool support for collaborative software development based on dependency analysis," in CollaborateCom, 2010, pp. 1-10. 
[9] J. T. Biehl, M. Czerwinski, G. Smith, and G. G. Robertson, "Fastdash: a visual dashboard for fostering awareness in software teams," in Proceedings of the SIGCHI Conference on Human Factors in Computing Systems, ser. CHI '07. New York, NY, USA: ACM, 2007, pp. 1313-1322.

[10] S. Hupfer, L.-T. Cheng, S. Ross, and J. Patterson, "Introducing collaboration into an application development environment," in Proc. of the 2004 ACM Conference on Computer Supported Cooperative Work, ser. CSCW '04. New York, NY, USA: ACM, 2004, pp. 21-24.

[11] C. R. de Souza, S. Quirk, E. Trainer, and D. F. Redmiles, "Supporting collaborative software development through the visualization of sociotechnical dependencies," in Proceedings of the 2007 international ACM conference on Supporting group work, ser. GROUP '07. New York, NY, USA: ACM, 2007, pp. 147-156.

[12] A. Sarma, L. Maccherone, P. Wagstrom, and J. Herbsleb, "Tesseract: Interactive visual exploration of socio-technical relationships in software development," in Proceedings of the 31st International Conference on Software Engineering, ser. ICSE '09. Washington, DC, USA: IEEE Computer Society, 2009, pp. 23-33.

[13] A. Borici, K. Blincoe, A. Schrter, G. Valetto, and D. Damian, "Proxiscientia: Toward real-time visualization of task and developer dependencies in collaborating software development teams." in CHASE. IEEE, 2012, pp. 5-11.

[14] M. Figueiredo and C. de Souza, "Wolf: Supporting impact analysis activities in distributed software development," in Cooperative and $\mathrm{Hu}$ man Aspects of Software Engineering (CHASE), 2012 5th International Workshop on, june 2012, pp. $40-46$.

[15] M. Conway, "How do committees invent," Datamation, vol. 14, no. 4, pp. 28-31, 1968.

[16] M. Cataldo, P. A. Wagstrom, J. D. Herbsleb, and K. M. Carley, "Identification of coordination requirements: implications for the design of collaboration and awareness tools," in Proceedings of the 2006 20th anniversary conference on Computer supported cooperative work, ser. CSCW '06. New York, NY, USA: ACM, 2006, pp. 353-362.

[17] G. Valetto, M. Helander, K. Ehrlich, S. Chulani, M. Wegman, and C. Williams, "Using software repositories to investigate socio-technical congruence in development projects," in Proceedings of the Fourth International Workshop on Mining Software Repositories, ser. MSR '07. Washington, DC, USA: IEEE Computer Society, 2007, pp. 25-.

[18] G. Madey, V. Freeh, and R. Tynan, "The open source software development phenomenon: An analysis based on social network theory," in Proceedings of the Americas Conference on Information Systems (AMCIS 2002), Dallas, Texas, 2002, pp. 1806-1813.

[19] T. Wolf, A. Schröter, D. Damian, L. D. Panjer, and T. H. D. Nguyen, "Mining task-based social networks to explore collaboration in software teams," IEEE Softw., vol. 26, no. 1, pp. 58-66, Jan. 2009.

[20] L. Jiang, K. M. Carley, and A. Eberlein, "Assessing team performance from a socio-technical congruence perspective," in ICSSP, 2012, pp. 160-169.

[21] T. W. Malone and K. Crowston, "The interdisciplinary study of coordination," ACM Comput. Surv., vol. 26, pp. 87-119, March 1994.

[22] C. Dorn and R. N. Taylor, "Analyzing runtime adaptability of collaboration patterns," in International Conference on Collaboration Technologies and Systems (CTS). Los Alamitos, CA, USA: IEEE Computer Society, 2012.

[23] S. Dustdar and T. Hoffmann, "Interaction pattern detection in process oriented information systems," Data Knowl. Eng., vol. 62, pp. 138-155, July 2007

[24] P. F. Xiang, A. T. T. Ying, P. Cheng, Y. B. Dang, K. Ehrlich, M. E. Helander, P. M. Matchen, A. Empere, P. L. Tarr, C. Williams, and S. X. Yang, "Ensemble: a recommendation tool for promoting communication in software teams," in Proceedings of the 2008 international workshop on Recommendation systems for software engineering, ser. RSSE '08. New York, NY, USA: ACM, 2008, pp. 2:1-2:1.

[25] D. W. McDonald and M. S. Ackerman, "Expertise recommender: a flexible recommendation system and architecture," in Proceedings of the 2000 ACM conference on Computer supported cooperative work, ser. CSCW '00. New York, NY, USA: ACM, 2000, pp. 231-240.

[26] D. Čubranić and G. C. Murphy, "Hipikat: recommending pertinent software development artifacts," in Proceedings of the 25th International Conference on Software Engineering, ser. ICSE '03. Washington, DC, USA: IEEE Computer Society, 2003, pp. 408-418.

[27] K. Blincoe, G. Valetto, and S. Goggins, "Proximity: a measure to quantify the need for developers' coordination," in Proceedings of the
ACM 2012 conference on Computer Supported Cooperative Work, ser. CSCW '12. New York, NY, USA: ACM, 2012, pp. 1351-1360.

[28] D. Schall, F. Skopik, and S. Dustdar, "Expert discovery and interactions in mixed service-oriented systems," IEEE Transactions on Services Computing, vol. 5, pp. 233-245, 2012.

[29] F. Skopik, D. Schall, and S. Dustdar, "Start trusting strangers? bootstrapping and prediction of trust," in Proceedings of the 10th International Conference on Web Information Systems Engineering, ser. WISE '09. Berlin, Heidelberg: Springer-Verlag, 2009, pp. 275-289.

[30] X. Zhao and L. Osterweil, "An approach to modeling and supporting the rework process in refactoring," in Software and System Process (ICSSP), 2012 International Conference on, june 2012, pp. $110-119$.

[31] X. Zhao, B. S. Lerner, L. J. Osterweil, E. R. Boose, and A. M. Ellison, "Provenance support for rework," in Proceedings of the 4th USENIX conference on Theory and Practice of Provenance, ser. TaPP'12. Berkeley, CA, USA: USENIX Association, 2012, pp. 14-14.

[32] R. Hebig, A. Seibel, and H. Giese, "Toward a comparable characterization for software development activities in context of mde," in Proc. of the 2011 Int. Conference on Software and Systems Process, ser. ICSSP' 11. New York, NY, USA: ACM, 2011, pp. 33-42.

[33] K. A. Kedji, R. Lbath, B. Coulette, M. Nassar, L. Baresse, and F. Racaru, "Supporting collaborative development using process models: An integration-focused approach," in ICSSP, 2012, pp. 120-129.

[34] T. Moser, S. Biffl, W. D. Sunindyo, and D. Winkler, "Integrating production automation expert knowledge across engineering domains," IJDST, vol. 2, no. 3, pp. 88-103, 2011.

[35] A. Egyed, "Automatically detecting and tracking inconsistencies in software design models," IEEE Transactions on Software Engineering, vol. 37, no. 2, pp. 188-204, 2011.

[36] J. C. Grundy, J. G. Hosking, and W. B. Mugridge, "Inconsistency management for multiple-view software development environments," IEEE Trans. on Software Eng., vol. 24, no. 11, pp. 960-981, 1998.

[37] Y. Xiong, Z. Hu, H. Zhao, H. Song, M. Takeichi, and H. Mei, "Supporting automatic model inconsistency fixing," in 7th Joint Meeting of the European Software Engineering Conference (ESEC) and the ACM SIGSOFT International Symposium on Foundations of Software Engineering (FSE), Amsterdam, The Netherlands, August 2009, pp. 315-324.

[38] R. F. Paige, P. J. Brooke, and J. S. Ostroff, "Metamodel-based model conformance and multiview consistency checking," ACM Transactions on Software Engineering and Methodology, vol. 16, no. 3, 2007.

[39] M. Sabetzadeh, S. Nejati, S. M. Easterbrook, and M. Chechik, "Global consistency checking of distributed models with tremer+," in 30th International Conference on Software Engineering (ICSE), Leipzig, Germany, May 2008, pp. 815-818.

[40] M. Lanza, H. Gall, and P. Dugerdil, "Evospaces: Multi-dimensional navigation spaces for software evolution," in 13th European Conference on Software Maintenance and Reengineering (CSMR), Kaiserslautern, Germany, March 2009, pp. 293-296.

[41] A. Egyed, E. Letier, and A. Finkelstein, "Generating and evaluating choices for fixing inconsistencies in uml design models," in 23rd IEEE/ACM International Conference on Automated Software Engineering (ASE), L'Aquila, Italy, September 2008, pp. 99-108.

[42] M. Kamalrudin, J. C. Grundy, and J. G. Hosking, "Managing consistency between textual requirements, abstract interactions and essential use cases," in 34th Annual IEEE Int. Computer Software and Applications Conf. (COMPSAC), Seoul, Korea, July 2010, pp. 327-336.

[43] C. Nentwich, W. Emmerich, and A. Finkelstein, "Consistency management with repair actions," in 25th International Conference on Software Engineering (ICSE), Portland, Oregon, USA, May 2003, pp. 455-464.

[44] A. Egyed, "Fixing inconsistencies in uml design models," in 29th International Conference on Software Engineering (ICSE), Minneapolis, MN, USA, May 2007, pp. 292-301.

[45] L. C. Briand, Y. Labiche, and L. O'Sullivan, "Impact analysis and change management of uml models," in 19th Int. Conf. on Software Maintenance (ICSM) Amsterdam, The Netherlands, September 2003, pp. 256-265.

[46] J. Cabot and E. Visser, Eds., Proceedings of the Int. Conf. on the Theory and Practice of Model Transformations (ICMT). Springer LNCS, 2011.

[47] S. Bohner and R. Arnold, Software Change Impact Analysis. IEEE Computer Society Press, Los Alamitos, California, 1996.

[48] A. Biere, M. Heule, H. van Maaren, and T. Walsh, Eds., Handbook of Satisfiability, ser. Frontiers in Artificial Intelligence and Applications, vol. 185. IOS Press, 2009. 